\title{
Hypertriglyceridemia: An indicator of poor prognosis in acute pancreatitis - A hospital based study
}

\author{
Mosin Mushtaq ${ }^{1,2}$, Abid H. Wani' ${ }^{2}$, Faud Sadiq ${ }^{2}$, Mudasir Mushtaq ${ }^{3}$, Sameena Tabassum ${ }^{4}$, Taiba Mudasir ${ }^{3}$ \\ ${ }^{1}$ Department of General Surgery, Sheri Kashmir Institute of Medical Sciences, Srinagar, J \& K, ${ }^{2}$ Department of General Surgery, Government \\ Medical College, Srinagar, J \& K., ${ }^{3}$ Department of Pediatrics, Royal Children Hospital, Melbourne, Australia, ${ }^{4}$ Department of Endodontics, \\ Saraswati Dental College, Lucknow, Uttar Pradesh
}

\section{A B S TR A C T}

\begin{abstract}
Background and study aims: Hypertriglyceridemia can be a primary cause for acute pancreatitis or secondary to other factors prior to the increase of lipid levels, or both. The aim of our study was to assess the severity of acute pancreatitis with elevation in serum triglyceride levels and report the outcomes of our series. Patients and methods: One hundred twenty-six patients of acute pancreatitis were admitted within 72 hours of onset of symptoms, out of whom 26 patients were excluded during the course of study due to preexisting comorbidities. 100 patients in the study population were divided into group $A$ having serum triglyceride levels $\geq 500 \mathrm{mg} / \mathrm{dl}$ ( $\mathrm{n}=30$ ) and group $B$ having $<500 \mathrm{mg} / \mathrm{dl}(\mathrm{n}=70$ ) at the time of admission. Results: The mean age of group A was similar to group B (50.2 \pm 17.1 vs $49.26 \pm 17.2$ years; $p=0.860$ ). Most common etiological factor of acute pancreatitis was found to be gall stones (56\%) in both groups. Ranson's score at admission in group A was $2.93 \pm 0.22$ and in group B it was $1.34 \pm 0.99$. Mortality below two weeks was noted in $(12 / 30,40 \%)$ of group $A$ and $(6 / 70,8 \%)$ of group B patients, which was statistically significant, $(p=0.015)$. Conclusions: The final conclusion of this study was that patients of acute pancreatitis with elevated triglyceride levels form a morbid group and these should be monitored aggressively for the development of any complications.
\end{abstract}

Key words: Hypertriglyceridemia, Acute Pancreatitis, Necrosis, Pseudocyst

\section{INTRODUCTION}

Hypertriglyceridemia is defined as increase in fasting plasma triglyceride levels typically above the $95^{\text {th }}$ percentile for age and sex, although additional quantitative or qualitative lipoprotein abnormalities can also be present. ${ }^{1,2}$ Although a serum triglyceride level of more than $1000 \mathrm{mg} / \mathrm{dL}$ is needed to precipitate acute pancreatitis, the reduction of which to well below $1000 \mathrm{mg} / \mathrm{dL}$ may effectively prevent further episodes. ${ }^{3}$ Hypertriglyceridemia is one of the known causes of acute pancreatitis, seen in 1.3-3.8\% of patients as a primary etiological factor or secondary to other factors prior to the increase of lipid levels but constituting clinically relevant proportion of cases..$^{3-7}$ Some patients can develop acute pancreatitis when their fasting triglyceride concentration is $500-1000 \mathrm{mg} / \mathrm{dl}$, its risk becomes clinically significant when fasting measurements exceed $1000 \mathrm{mg} / \mathrm{dl}$, a level at which chylomicrons are present. ${ }^{6,7}$

It has been seen that triglyceridemia would worsen pancreatic injury induced by acute pancreatitis when it reaches $500 \mathrm{mg} / \mathrm{dl}$ or more, thus playing an important role in predisposing mild pancreatitis to the vicious episode. ${ }^{8}$ Extreme elevation of triglycerides in acute pancreatitis patients with familial hypertriglyceridemia can cause the so-called hyperlipidemic abdominal crisis. ${ }^{9}$ However, severe acute pancreatitis patients with hypertriglyceridemia can have pancreatic necrosis, pseudo cysts, abscesses and other complications that can be seen in other types of pancreatitis with a different clinical course from other forms of acute pancreatitis. ${ }^{10}$ The correlation 
between hypertriglyceridemia and the severity of acute pancreatitis is still uncertain. The aim of the present study was to analyse the effect of hypertriglyceridemia on the outcome of severe acute pancreatitis in view of morbidity and mortality.

\section{MATERIALS AND METHODS}

This present study was a hospital based prospective study conducted in the Department of General Surgery, Government Medical College, Srinagar, in collaboration with the department of Biochemistry over a period of one and a half years from June 2012 to December 2013. One hundred twenty-six patients of acute pancreatitis who reported to surgical department of this hospital within 72 hours of onset of symptoms were admitted, out of whom 26 patients were excluded during the course of study. Exclusion criteria were

1. Cases of mild acute pancreatitis

2. Patients with pre-existing cardiovascular, renal or hepatic dysfunction

3. Contrast allergy to CECT.

100 patients enrolled in the study were divided into two groups according to admission triglyceride levels. Group A having serum triglyceride levels $\geq 500 \mathrm{mg} / \mathrm{dl}(\mathrm{n}=30)$ and Group B having $<500 \mathrm{mg} / \mathrm{dl}(\mathrm{n}=70)$. Hypertriglyceridemia was present in all patients $(>150 \mathrm{mg} / \mathrm{dl})$.

After a detailed history and clinical examination, routine investigations and pancreatic profile was sent. X-ray chest and abdominal ultrasonography was done on routine basis. Contrast enhanced computed tomography abdomen was done typically after 72 hours of admission when it is optimum to rule out pancreatic necrosis and properly delineate areas of necrosis. Estimation of serum triglyceride levels was carried out within 24 hours of admission in department of biochemistry with (Hitachi 912) auto analyser. The serum sample was analysed by computer based software system of the autoanalyser to get the value of serum triglycerides. The patients were managed on the standardized protocols of severe acute pancreatitis in intensive care unit setting.

The sex, age, etiology, admission biochemical parameters and incidence of complications including acute respiratory distress syndrome, hypoxemia, renal failure, shock, encephalopathy, multiple organ dysfunction syndrome, local complications, hospital stay and mortality were evaluated and confirmed by single investigator. The Ranson's score and admission Balthazar's computed tomography score were calculated by a single investigator. Standard operative procedure if any remained percutaneous drainage, laparotomy and pancreatic necrosectomy and closed lavage.

\section{Statistical analysis}

All data were expressed as means \pm standard deviation. Quantitative data between two groups were compared using paired or unpaired Student t-test for parametric data and the Mann-Whitney $U$ test for nonparametric data. Categorical data were compared with Fischer's exact test and $\chi^{2}$ test. A two tailed $p$ value of $<0.05$ was considered significant. Statistical analysis was done with Statistical package for the Social Sciences for windows.

\section{RESULTS}

A total of 100 patients were enrolled in the study. 30 patients in the group A having serum triglyceride levels $\geq 500 \mathrm{mg} / \mathrm{dl}$ and 70 patients in the group B with serum triglyceride levels $<500 \mathrm{mg} / \mathrm{dl}$ at the time of admission. There was no significant difference between two groups in age and sex. The mean age of group $\mathrm{A}$ was similar to group B (50.2 \pm 17.1 vs $49.26 \pm 17.2$ years; $p=0.860)$. Most common etiological factor of acute pancreatitis was found to be gall stones (56\%) in both groups, followed by worm induced pancreatitis $(20 \%)$, alcohol $(10 \%)$, hypertriglyceridemia $(2 \%)$ and other causes $(12 \%)$. Clinically in both the groups pain was the most common symptom while as epigastric tenderness was the most common sign. In all the patients ultrasonography of abdomen done at the time of admission revealed features suggestive of acute pancreatitis. In our study, Ranson's score at admission in group A was $2.93 \pm 0.22$ and in group B it was $1.34 \pm 0.99$. The difference in means was statistically significant, $(\mathrm{p} \leq 0.001)$. Computed tomography severity index scores in the group A was $8.80 \pm 1.01$ and in group B it was $6.86 \pm 1.21,(\mathrm{p} \leq 0.001)$. There was a significant difference between the two groups in view of admission time biochemical parameters and the occurance of complications (systemic and local) during the hospital stay. Table 1 and 2 summarizes these events in both the groups.

Among the group A $(8 / 30,27 \%)$ patients underwent necrosectomy and among group B (10/70,14\%) patients underwent necrosectomy. Four patients in the group A and four patients in group B expired after surgical intervention, $(p=0.420)$. In our study mean hospital stay for group A and group B patients was 22.85 days and 21.6 days, $(\mathrm{p}=0.769)$. The overall mortality in our study was $(28 / 100,28 \%)$ out of which $(64 \%)$ of the patients died within two weeks of admission. Late mortality was noted in $(36 \%)$ of patients. Mortality below two weeks was noted in $(12 / 30,40 \%)$ of group A and $(6 / 70,8 \%)$ of group B patients, which was statistically significant, $(p=0.015)$. 


\begin{tabular}{|c|c|c|c|c|c|}
\hline \multirow[t]{2}{*}{ Parameter } & \multicolumn{2}{|c|}{ Group A $(n=30)$} & \multicolumn{2}{|c|}{ Group B (n=70) } & \multirow[t]{2}{*}{$p$ value } \\
\hline & Mean & Standard deviation & Mean & Standard deviation & \\
\hline 1. Serum amylase & $625 \mathrm{U} / \mathrm{L}$ & 358.75 & $758 \mathrm{U} / \mathrm{L}$ & 171.95 & 0.080 \\
\hline 2. Serum lipase & $253 \mathrm{U} / \mathrm{L}$ & 102.20 & $359 \mathrm{U} / \mathrm{L}$ & 90.44 & $\leq 0.001$ \\
\hline 3. Serum albumin & $3.29 \mathrm{~g} / \mathrm{L}$ & 0.218 & $3.51 \mathrm{~g} / \mathrm{L}$ & 0.353 & 0.030 \\
\hline 4. Serum calcium & $7.6 \mathrm{mg} / \mathrm{dl}$ & 0.228 & $8.1 \mathrm{mg} / \mathrm{dl}$ & 0.662 & 0.003 \\
\hline 5. Serum blood sugar & $223.4 \mathrm{mg} / \mathrm{dl}$ & 55.869 & $180.6 \mathrm{mg} / \mathrm{dl}$ & 37.714 & 0.003 \\
\hline 6. Serum triglycerides & 645 mg/dl & 158.912 & 242 mg/dl & 74.907 & $\leq 0.001$ \\
\hline
\end{tabular}

\begin{tabular}{|c|c|c|c|}
\hline Parameter & $\begin{array}{c}\text { Group A } \\
(n=30)\end{array}$ & $\begin{array}{c}\text { Group B } \\
(n=70)\end{array}$ & $p$ value \\
\hline 1. Hypoxemia & $20(67 \%)$ & $22(32 \%)$ & 0.045 \\
\hline 2. Pleural effusion & $18(60 \%)$ & $36(52 \%)$ & 0.758 \\
\hline *3. ARDS & $20(67 \%)$ & $34(48 \%)$ & 0.335 \\
\hline †4. Renal failure & $20(67 \%)$ & $14(20 \%)$ & 0.003 \\
\hline ‡5. Shock & $18(60 \%)$ & $18(26 \%)$ & 0.028 \\
\hline 6. Encephalopathy & $16(53 \%)$ & $14(20 \%)$ & 0.078 \\
\hline §7. MODS & $20(67 \%)$ & $14(20 \%)$ & 0.003 \\
\hline 8. Necrosis & $16(53 \%)$ & $16(23 \%)$ & 0.049 \\
\hline 9. Pancreatic abscess & $4(13 \%)$ & $4(6 \%)$ & 0.574 \\
\hline 10.Pseudocyst & $4(13 \%)$ & $4(6 \%)$ & 0.574 \\
\hline
\end{tabular}

*Acute respiratory distress syndrome $\left(\mathrm{PaO}_{2} / \mathrm{FiO}_{2}<200\right)$. ${ }^{\text {TRenal failure (serum creatinine }}$ of $>2 \mathrm{mg} / \mathrm{dl}$ despite adequate rehydration). ${ }^{{ }^{*}} \mathrm{~S} h o c k, \mathrm{MAP}<70 \mathrm{mmHg}$, requiring lonotropic support. sMulti organ dysfunction syndrome (SIRS with $\geq 0 n e$ vital organ dysfunction)

\section{DISCUSSION}

Hypertriglyceridemia associated severe acute pancreatitis is an uncommon, but potentially life-threatening disease. Hypertriglyceridemia may be primary in origin (hereditary or sporadic genetic disorder of metabolism) or secondary (associated with an identifiable disease or condition and is reversible with control or eradication of that disease or condition) or both. Although the exact pathogenesis of hypertriglyceridemia associated acute pancreatitis is not clear, it is thought to result from toxic injury to acinar cells and capillary endothelia by excessive free fatty acids from hydrolysis of triglycerides. Severe acute pancreatitis patients with hypertriglyceridemia ( $>500 \mathrm{mg} / \mathrm{dl}$ ) had a higher incidence of hypoalbuminaemia and hypocalcemia and a higher level of admission serum glucose, which may be associated with the aggravation of hypertriglyceridemia, resulting from severe stress response and metabolic disorders. The role of hypertriglyceridemia in modulating disease course of acute pancreatitis is still controversial but it was reported that hypertriglyceridemia is independently associated with the severity of acute pancreatitis and plays a role in the aggravation of acute necrotizing pancreatitis. The results of our study show that the incidence of admission hypocalcaemia and hypoalbuminaemia are a predictable index of severe acute pancreatitis. Ranson's score at admission and computed tomography severity index scores were higher in the hypertriglyceridemia group than in the control group. The complications such as renal failure, shock and infection, MODS and the mortality were higher in the hypertriglyceridemia group than in the control group, indicating that hypertriglyceridemia aggravates severe acute pancreatitis leading to systemic complications and a high mortality rate.

In our study, Ranson's score and computed tomography severity index score between the two groups was statistically significant, $(p<0.05)$. We had higher mortality in group A (in which triglyceride levels were $\geq 500 \mathrm{mg} / \mathrm{dl}$ ) and this goes in consistant with higher Ranson's score and computed tomography severity index score in this group. Vrein PW et al. ${ }^{11}$ and Chisty IA et al. ${ }^{12}$ noted a morbidity of $100 \%$ in patients with CTSI of 7-10, favouring results of our study. The levels of biochemical parameters like serum amylase, lipase, calcium, blood sugar, albumin and triglycerides between the two groups were statistically significant. These parameters were more unfavourable in group $\mathrm{A}$ as compared to group $\mathrm{B}$ in consistant with other reported studies like Navarro S et al. ${ }^{13}$ and Deng LH et al. ${ }^{14}$ Harrison DA et al. ${ }^{15}$ in their study suggested biochemical variables having strong relation with the outcome including serum calcium level in decreasing order.

Pleural effusion and acute respiratory distress syndrome were the most common complications present in both the groups. Pulmonary dysfunction ranging from hypoxemia to acute respiratory distress syndrome is one of the most important systemic manifestations of severe acute pancreatitis occurring in $30-50 \%$ of patients. ${ }^{16,17}$ In our study the complications like hypoxia, pleural effusion, shock, acute respiratory distress syndrome, encephalopathy, renal failure and multi organ dysfunction syndrome occurred more in group A patients where triglyceride levels were more than $500 \mathrm{mg} / \mathrm{dl}$. Our results resemble the studies conducted by Raghu et al., ${ }^{18}$ Lankisch et al., ${ }^{19}$ Deng LH et al., ${ }^{14}$ Fortson MR et al. ${ }^{4}$ and Anderson $\mathrm{F}$ et al. ${ }^{20}$ In our study infective necrosis was present in $32 \%$ of the patients, but incidence rate of infective necrosis was higher in group A (53\%) than in group B $(22 \%), p<0.05$. However rates of pancreatic abscesses and pseudocyst formation between the two 
groups were statistically insignificant. Fortson MR et al. ${ }^{4}$ in their study reported an incidence of abscesses in 13\% of patients which is similar to our results. The mean hospital stay in our study is nearly same as documented by Chisty IA ${ }^{12}$ in his work which had an average hospital stay of 25.12 days for severe pancreatitis. The overall mortality in our study was more for group A patients who had triglyceride levels more than $500 \mathrm{mg} / \mathrm{dl}$ which is in line with the reported literature world over. ${ }^{14,21,22}$ Our study is in concordance with most of the studies world over.

\section{CONCLUSION}

The final conclusion of this study is that patients of acute pancreatitis with elevated triglyceride levels form a morbid group and these should be monitored aggressively for the development of any complications.

\section{REFERENCES}

1. Hegele RA. Monogenic dyslipidemias: window on determinants of plasma lipoprotein metabolism Am J Hum Genet 2001; 69: 1161.

2. Hodis HN, Mack WJ, Krauss RM and Alaupovic P. Pathophysiology of triglyceride-rich lipoproteins in atherothrombosis: clinical aspects. Clin Cardiol 1999; 22:15-20.

3. Toskes PP. Hyperlipidemia pancreatitis. Gastroenterol Clin North Aml 1990; 19: 783-791.

4. Fortson MR, Freedman SN and Webster PD. Clinical assessment of hyperlipidemic pancreatitis. Am J Gastroenterol 1995; 90: 2134-2139.

5. Santamarina Fojo S. The familial chylomicronemia syndrome. Endocrinol Metab Clin North Am 1998; 27: 551-567.

6. Athyros VG, Giouleme OI, Nikolaidis NL, Vasiliadis TV, Bouloukos VI, Kontopoulos AG, et al. Long term follow up of patients with acute hypertriglyceridemia induced pancreatitis. J Clin Gastroenterol 2002; 34: 472-475.

7. Yadav $D$ and Pitchumoni CS. Issues in hyperlipidemic pancreatitis. J Clin Gastroenterol 2003; 36: 54-62.

8. Oh RC and Lanier JB. Management of hypertriglyceridemia. Am Fam Physician 2007; 75: 1365-1371.
9. Miller A, Lees RS, McCluskey MA and Warshaw AL. The natural history and surgical significance of hyperlipemic abdominal crisis. Ann Surg 1979; 190: 401-408.

10. Balachandra S, Virlos IT, King NK, Siriwardana HP, France MW and Siriwardena AK. Hyperlipidaemia and outcome in acute pancreatitis. Int J Clin Pract 2006;60: 156-159.

11. Vriens PW, Linde $\mathrm{P}$ and Warmerdon PE. Computed tomography severity index is an early prognostic tool for acute pancreatitis. J Amern Col Surgns 2004; 201: 497-502.

12. Chisty IA, Vaqar B, Sajida $P$ and Zishan $H$. Role of CT in acute pancreatitis and its complications among age groups. Journal of Pak Med Asso 2005; 55:431-435.

13. Navarro S, Cubiella J, Feu F, Zambon D, Fernandez-Cruz $L$ and Ros E. Hypertriglyceridemic acute pancreatitis. Is its clinical course different from lithiasic acute pancreatitis?. Med Clin (Barc) 2004; 123: 567-570.

14. Deng LH, Xue P, Xia Q, Yang XN and Wan MH. Effect of admission Hypertriglyceridemia on the episodes of severe acute pancreatitis. World J Gastroenterol 2008; 14 (28): 4558-4561.

15. Harrison DA, D'Amico $G$ and Singer M. The Pancreatitis Outcome Prediction (POP) Score: a new prognostic index for patients with severe acute pancreatitis. Crit Care Med. 2007; 35 (7): 1703-1708.

16. Ranson JH, Roses DF and Fink SD. Early respiratory insufficiency in acute pancreatitis. Ann Surg 1973; 178: 75-79.

17. Napolitano LM. Pulmonary consequences of acute pancreatitis: Critical role of the neutrophil. Crit Care Med 2002; 30: 2158-2159.

18. Raghu MG, Wig JD, Kochhar R, Gupta D, Gupta R, Yadav TD, et al. Lung Complications in Acute Pancreatitis. JOP 2007; 8 (2): 177-185.

19 Lankisch PG, Droge M and Becher R. Pleural effusions: a new negative prognostic parameter for acute pancreatitis. Am J Gastroenterol 1994; 89: 1849-1851.

20. Anderson F, Thomson SR, Clarke DL and Buccimazza I. Dyslipidaemic pancreatitis clinical assessment and analysis of disease severity and outcomes. Pancreatology 2009; 9: 252-257.

21. Shah SSH, Ansari MA and Ali S. Early prediction of severity and outcome of acute severe pancreatitis. Pak J Med Sci 2009; 25: 619-623.

22. Murad M, Hazem A, Coto-Yglesias F, Dzyubak S, Gupta S, Bancos I, et al. The association of hypertriglyceridemia with cardiovascular events and pancreatitis: a systematic review and meta-analysis. BMC Endocrine Disorders 2012; 12: 2.

\footnotetext{
Authors Contribution:

MM - Conceptualized study and prepared first draft of manuscript, AHW - Participated in design of study and revised draft of manuscript, FS - Performed biochemical analysis needed for study, MM - Correction of the manuscript and editing, ST - Statistical analysis of data, TM - Evaluation and Literature Collection
}

Source of Support: Government Hospital for people, no particular source of funding. Conflict of Interest: None declared. 